\title{
Serum Levels of Seven General Cytokines in Acute Brucellosis Before and After Treatment
}

\author{
Yunxia Tang ${ }^{1,2, *}$ \\ Chenjie $\mathrm{Ma}^{\mathrm{l}, 2, *}$ \\ Huali Sun ${ }^{3}$ \\ Siyuan Yang $\mathbb{D}^{1,2}$ \\ Fengting $\mathrm{Yu}^{1,2}$ \\ Xingwang $\mathrm{Li}^{1,2}$ \\ Linghang Wang ${ }^{1,2}$
}

\begin{abstract}
'Beijing Ditan Hospital, Capital Medical University, Beijing, People's Republic of China; ${ }^{2}$ Clinical Center for HIV/AIDS,

Capital Medical University, Beijing,

People's Republic of China; ${ }^{3}$ Department of Infectious Diseases, The Affiliated

Hospital of Qingdao University, Qingdao,

People's Republic of China
\end{abstract}

*These authors contributed equally to this work
Correspondence: Linghang Wang

Beijing Di Tan Hospital, Capital Medical University, Beijing, People's Republic of China

Tel/Fax +86-10-84323103

Email linghang.wang@ccmu.edu.cn
Purpose: Human brucellosis is the most common bacterial zoonosis globally that poses a severe health threat. Despite the availability of antibiotic therapy for human brucellosis, its tendencies of chronicity and persistence may lead to severe debilitating and disabling conditions in patients. Comprehensive understanding of the immune response in brucellosis will be helpful in improving the treatment strategies. In this study, we measured serum levels of T helper cell type 1 (Th1), Th2, and Th17 cytokines in patients with acute brucellosis before and after treatment.

Patients and Methods: Overall, 30 patients with acute brucellosis from the Beijing Di Tan Hospital and 26 healthy controls were enrolled in this study. All the patients received a 6-week therapy regimen comprising ceftriaxone, doxycycline, and rifampicin. Serum samples were collected from patients with acute Brucella infection and healthy controls before and after treatment. Serum seven cytokine levels of Th1 (IL-2, IFN- $\gamma$, and TNF- $\alpha$ ), Th2 (IL-4, IL-6, IL-10), and Th17 (IL-17A) were measured using cytometric bead array.

Results: In patients with acute infection, the IL-2, IFN- $\gamma$, and IL-10 levels were significantly increased compared with those in healthy controls $(\mathrm{P}<0.001)$. After treatment, IL-2, IFN- $\gamma$, and IL-10 levels significantly decreased $(\mathrm{P}<0.05)$ and the TNF- $\alpha$ level significantly increased compared with the corresponding baseline levels and those in healthy controls $(\mathrm{P}$ $<0.05$ ). Furthermore, the IFN- $\gamma$, IL-4, and IL-10 levels were higher in patients after treatment than in healthy controls $(\mathrm{P}<0.05)$. IL-2 and IL-6 levels exhibited a positive correlation with the $\mathrm{C}$-reactive protein (CRP) level in acute brucellosis $(\mathrm{P}<0.05)$.

Conclusion: Levels of most serum Th1 and Th2 cytokines were simultaneously increased in acute infection, followed by reduction in the corresponding cytokine levels and residual cytokine response during treatment. This residual immune response could represent a therapeutic opportunity that may improve the long-term clinical outcomes in patients with acute brucellosis after treatment.

Keywords: brucellosis, acute infection, Th1, Th2, cytometric bead array, cytokines

\section{Introduction}

Human brucellosis remains one of the most common zoonoses worldwide and is a major public health issue in many developing countries. ${ }^{1}$ Because of nonspecific clinical manifestations, brucellosis is often under-diagnosed and under-reported, particularly in regions with poor healthcare access. ${ }^{2}$ Furthermore, since human brucellosis has tendencies of chronicity and persistence, the disease can cause severe debilitating and disabling conditions in patients, affecting all the organs. ${ }^{3}$ General principles of the clinical management of brucellosis include prompt and adequate combination antibiotic therapy. To reduce relapses and chronicity, current strategies for brucellosis treatment advocate prolonged duration of treatment 
combined with antibiotic regimens (such as doxycycline and rifampin) that play a role in the acidic intracellular environment. ${ }^{4}$ Despite the availability of satisfactory treatments against brucellosis, treatment failures and relapses are inevitable to some extent. ${ }^{5}$ Thus, further studies are urgently needed to develop optimum treatment regimens to control this disease.

Brucellosis is caused by facultative intracellular bacteria belonging to Brucella genus that can survive and multiply within phagocytic cells. After infecting the host, the bacteria become lurked in the cells of the reticuloendothelial system and then invade and modulate the host immune system. ${ }^{6}$ Interaction of Brucella with the human immune system is critical for infection outcomes, ${ }^{6}$ which have been the focus of many studies and debates. Host immune protection against Brucella depends on cellmediated immunity, which mainly involves activated antigen-presenting cells (macrophages and dendritic cells), $\mathrm{CD}^{+} \mathrm{T}$ helper cells (Th), and $\mathrm{CD} 8^{+}$cytotoxic $\mathrm{T}$ cells (Tc). ${ }^{7,8}$ Macrophage antigen presentation and activation of T-cell proliferation result in differentiation and secretion of cytokines to harmonise the immune response, thereby determining pathogen clearance or disease progression. ${ }^{7}$ Clinical features of human brucellosis are remarkably heterogeneous, and infection control is largely dependent on the host immune status. ${ }^{9}$

It is well established that the expression of cytokines is necessary for a normal protective function of the immune response. The characteristics of immune responses of brucellosis have been studied in animal models and cells. ${ }^{10-15}$ Generally, the infection control and recovery in animals require Th1-type response characterised by the expression of IFN- $\gamma$ and IL-2, whereas the Th2 response, characterised by IL-4 and IL-10 expressions, determines the susceptibility to experimental infections with Brucella and poor prognosis. ${ }^{10-13}$ In human brucellosis, increasing evidence suggests that infection induced by Brucella disrupts cytokine expression and function of the $\mathrm{CD} 4^{+} \mathrm{Th}$ and $\mathrm{CD} 8^{+}$cytotoxic T cells and that infection progression is mainly associated with Th2 cytokines. ${ }^{16,17}$ In particular, TNF- $\alpha$, IFN- $\gamma$, IL-2, and IL10 levels are affected during Brucella infection. ${ }^{16,18,19}$ Additionally, the level of these cytokine indicates the status of the immune response and disease progression. However, the role of $\mathrm{Th} 1 / \mathrm{Th} 2 / \mathrm{Th} 17$ cytokines in acute brucellosis infection and treatment still remains partially defined or debated. ${ }^{16-20}$
In this prospective cohort study, we enrolled 30 patients with acute brucellosis and estimated changes in the serum levels of seven general Th1/Th2/Th17 cytokines during treatment by using cytometric bead array (CBA); healthy controls matched by age and sex were also included in the study. Furthermore, we followed up the treated individuals for 6 months. Our aim was to evaluate the immune response in the early stage of human brucellosis and to identify promising candidate biomarkers for treatment monitoring of this infection.

\section{Patients and Methods Patient Registration and Selection Criteria}

A total of 30 patients with acute brucellosis were recruited from the Department of Infectious Disease of Beijing Ditan Hospital, Capital Medical University, from April 2018 to May 2019. All the patients were treated according to the therapeutic regimen comprising ceftriaxone (at $2 \mathrm{~g}$ /day for 2 weeks), doxycycline (at $100 \mathrm{mg}$ twice daily for 6 weeks), and rifampin (at 600-900 mg/day for 6 weeks). ${ }^{21}$ The diagnosis of human brucellosis was presumed based on the standard agglutination test (SAT) titre $\geq 1 / 100 \quad(2+),{ }^{4}$ relevant signs and symptoms (including fever, sweating, and arthralgias), and relevant epidemiological exposure (including consumption of unpasteurised dairy products and livestock exposure). A definitive diagnosis of brucellosis was established based on isolation of the genus Brucella from blood samples, body fluids, or tissues and/or through the positive serological test. The diagnostic criteria and clinical stage of brucellosis were in strict accordance with the expert consensus issued by China in 2017 for the diagnosis and treatment of brucellosis. ${ }^{4}$ Patients whose duration of disease was less than 6 months were considered as having acute infection. The exclusion criteria were as follows: 1 . individuals with respiratory insufficiency; autoimmune, endocrine and metabolic, blood, or nervous system diseases; liver and kidney diseases; or cancer; 2. patients taking immunosuppressive and immunomodulatory agents; 3 . patients who had received antibiotic treatment 3 months prior to the study; 4 . breast-feeding women; and 5. individuals aged less than 18 years. Overall, 26 sex- and age-matched healthy controls were enrolled from the same region as the patients with Brucella infection. 


\section{Data and Samples Collection}

Demographic, clinical, and laboratory characteristics (including age, gender, Brucella transmission route, symptoms and signs, time from symptoms, and diagnostic tests) were recorded. Serum was separated from blood obtained from the patients and healthy controls before antibiotic therapy and at 6 weeks after initiation of treatment. All serum samples were stored at $-80^{\circ} \mathrm{C}$ until use.

\section{General Inflammatory Marker Measurements}

Circulating levels of C-reactive protein (CRP) were tested in duplicates by using specific ELISA kits (R\&D Systems, Inc., Minneapolis, USA), according to the manufacturer's instructions. ${ }^{22}$ We measured the white blood cell (WBC) count and erythrocyte sedimentation rate (ESR) by using standard laboratory methods.

\section{CBA for the Measurement of Cytokines}

The Cytometric Bead Array Human Th1/Th2/Th17 Cytokine kit (BD Bioscience, San Jose, California) was used to quantitatively measure the IL-2, IL-4, IL-6, IL-10, TNF- $\alpha$, IFN- $\gamma$, and IL-17A levels in serum samples, following instruction of the manufacturer. ${ }^{23}$ Samples were measured on the BD FACSCalibur Flow Cytometer (BD Biosciences, San Jose, CA, USA) and data analysis was performed using BD FCAP ArrayTM Software (BD, US).

\section{Statistical Analysis}

Data are expressed as the median value and interquartile range (IQR). SPSS software 17.0 and GraphPad Prism were used for statistical analyses. Mann-Whitney $U$-test was used to compare the patients and healthy controls. Wilcoxon paired test was used for comparison between the observations before and after treatment for the same individuals. Spearman rank correlation test was used to quantify the association between Th1/Th2/Th17 cytokine levels and general inflammatory biomarkers in the patients. All tests were two-tailed, and P-values $<0.05$ were considered statistically significant.

\section{Results}

\section{Demographic and Clinical Characteristics of Patients with Acute Brucellosis}

The participant characteristics are shown in Table 1. Overall, 30 patients [21 (70\%) men] with acute brucellosis were included; the average age of the participants was 42 years (range: $21-65$ years). We recruited 26 healthy controls [19 (73\%) men], with an average age of 44 years (range: 24-65 years). No significant difference was found in terms of sex and age between the patient and healthy control groups $(\mathrm{P}>0.05)$. The most common symptoms of the patients were fever (86.67\%), sweating (73.33\%), arthralgias (46.67\%), and low back pain (46.67\%). Disease duration in all these patients was less than 10 weeks. The positive rate of blood culture was $43.33 \%$ (13/30); the positive rate for the Rose-Bengal test was $100 \%$, and the serum agglutination titre was $\geq 1 / 160$ in all enrolled subjects. In patients with acute brucellosis, the CRP level and ESR were significantly increased $(\mathrm{P}<$ 0.001; Table 2). The healthy controls had negative RoseBengal test and normal inflammatory markers.

All the patients were treated according to the same antibiotic treatment regimen and followed up every 6 months. After the completion of treatment regimen, all the patients were clinically cured, as indicated by the gradual disappearance of symptoms and signs and significant decrease in the level of general inflammatory parameters $(P<0.001$; Table 2$)$, which returned to normal. After 6 months of follow-up, no relapses were found.

\section{Serum ThI/Th2/ThI7 Cytokine Levels in Untreated Patients with Acute Brucellosis}

To identify the serum cytokine expression pattern in patients with acute brucellosis, we collected serum samples and measured the concentrations of 7 general Th1/ Th2/Th17 cytokines from 30 patients and 26 healthy controls. As shown in Figure 1 and Table 2, the levels of IL-2, IFN- $\gamma$, IL-4, IL-6, and IL-10 were significantly higher in patients with infection than in healthy controls $(\mathrm{P} \leq 0.01)$, and differences in the IL-2, IFN- $\gamma$, and IL-10 levels were significant $(\mathrm{P}<0.001)$. Additionally, the TNF- $\alpha$ and IL$17 \mathrm{~A}$ levels were not markedly different between the untreated patients and controls $(\mathrm{P}>0.05)$.

\section{Alteration in Serum Th I/Th2/Th I 7 Cytokine Levels in Patients with Acute Brucellosis During Treatment}

Antibiotic therapy was initiated in all 30 patients with acute brucellosis, and all of them were re-evaluated 6 weeks later (Figure 1 and Table 2). The CRP level and ESR were significantly declined after 6 weeks of treatment compared with those at baseline $(\mathrm{P}<0.001)$. IL-2, IFN- $\gamma$, 
Table I Characterization of Individuals with Acute Brucellosis and Healthy Controls

\begin{tabular}{|c|c|c|}
\hline Variable & Healthy Controls $n=26$ & Acute Brucella Infection $n=30$ \\
\hline Age (y), (mean [range]) & $44(24-65)$ & $42(21-65)$ \\
\hline Gender (M/F ratio) & $19 / 7$ & $21 / 9$ \\
\hline \multicolumn{3}{|l|}{ Mode of transmission, no. (\%) } \\
\hline Contact with infected animals & NA & $23(76.67)$ \\
\hline Consumption of unpasteurized dairy products & NA & $2(6.67)$ \\
\hline Consumption of undercooked meat of sheep & NA & $3(10.00)$ \\
\hline No obvious history of contact & NA & $2(6.67)$ \\
\hline \multicolumn{3}{|l|}{ Symptoms and signs, no. (\%) } \\
\hline Fevers & 0 & $26(86.67)$ \\
\hline Sweating & 0 & $22(73.33)$ \\
\hline Arthralgias & 0 & $14(46.67)$ \\
\hline Low back pain & 0 & $14(46.67)$ \\
\hline Weakness & 0 & $6(20.00)$ \\
\hline Chill & 0 & $8(26.67)$ \\
\hline Headaches & 0 & I (3.33) \\
\hline Dizziness & 0 & $2(6.67)$ \\
\hline Dyspepsia & 0 & $2(6.67)$ \\
\hline Abdominal pain & 0 & I (3.33) \\
\hline cough & 0 & $3(10.00)$ \\
\hline Testicular swelling and pain & 0 & I (3.33) \\
\hline Hepatomegaly & 0 & I (3.33) \\
\hline \multicolumn{3}{|l|}{ Time from symptoms, n (\%) } \\
\hline$<28$ days & NA & $28(93.33)$ \\
\hline $28-56$ days & NA & $2(6.67)$ \\
\hline \multicolumn{3}{|l|}{ Diagnostic tests, n (\%) } \\
\hline Positive Rose-Bengal test & 0 & $30(100)$ \\
\hline Positive Agglutination test (SAT) & NA & $30(100)$ \\
\hline Positive Blood culture & NA & $13(43.33)$ \\
\hline
\end{tabular}

Note: The SAT titre $\geq 1 / 160$.

Abbreviations: SAT, serum agglutination test; NA, not available.

and IL-10 levels were decreased during the treatment compared with the baseline levels, and the differences in IL-2 ( $\mathrm{P}=0.001)$, IFN- $\gamma(\mathrm{P}=0.003)$, and IL-10 $(\mathrm{P}=0.017)$ were significant. The IL-6 level showed a downward trend. Importantly, a marked increase in the TNF- $\alpha$ level was noted compared with that at baseline and in healthy controls $(\mathrm{P}<0.05)$. In addition, the IFN- $\gamma$, IL-4, and IL-10 levels were higher than normal $(\mathrm{P}<0.05)$, whereas the IL2, IL-6, and IL-17A levels were normalised after treatment $(\mathrm{P}>0.05)$. 
Table 2 Serum ThI/Th2/ThI7 Cytokine Levels and General Inflammatory Markers in Patients with Acute Brucellosis Pre- and PostTreatment and Healthy Controls

\begin{tabular}{|c|c|c|c|c|}
\hline Markers & $\begin{array}{l}\text { Healthy Controls }(n= \\
\text { 26) Median (IQR) }\end{array}$ & $\begin{array}{l}\text { Pre-Treatment }(n=30) \\
\text { Median }(I Q R)\end{array}$ & $\begin{array}{l}\text { Post-Treatment }(n=30) \\
\text { Median }(I Q R)\end{array}$ & $\begin{array}{l}\text { P-value (Pre-Treated vs } \\
\text { Post-Treated) }\end{array}$ \\
\hline \multicolumn{5}{|l|}{ cytokines } \\
\hline IL-2 (pg/mL) & $16.75(14.40-18.55)$ & $25.46(17.65-36.28) * *$ & $17.65(16.29-20.00)$ & 0.001 \\
\hline IL-4 (pg/mL) & $21.08(19.5 I-23.39)$ & $24.14(20.89-27.19) *$ & $22.62(21.85-25.84) * *$ & NS \\
\hline IL-6 (pg/mL) & $22.93(12.78-38.78)$ & $57.26(15.69-121.00) * *$ & $28.18(11.13-163.9)$ & NS \\
\hline IL-I0 (pg/mL) & $5.22(4.038-6.27)$ & $7.13(5.80-9.68) * *$ & $5.92(5.22-7.30) *$ & 0.017 \\
\hline TNF- $\alpha(p g / m L)$ & II.06 (7.67-35.98) & $9.72(7.67-20.65)$ & $22.33(11.77-59.35) *$ & 0.037 \\
\hline $\mathrm{IFN}-\gamma(\mathrm{pg} / \mathrm{mL})$ & $5.22(4.21-6.43)$ & $10.13(7.38-15.85) * *$ & $6.67(5.22-8.19) *$ & 0.003 \\
\hline IL-I7A (pg/mL) & $17.88(12.36-25.18)$ & $23.46(12.88-31.68)$ & $19.98(15.27-29.74)$ & NS \\
\hline \multicolumn{5}{|c|}{ Inflammatory markers } \\
\hline CRP (mg/L) & $0.35(0.09-1.05)$ & $3.63(1.79-6.92) * *$ & $0.32(0.13-0.81)$ & $<0.001$ \\
\hline ESR $(\mathrm{mm} / \mathrm{h})$ & $12.90(8.75-14.13)$ & 35.15 (20.40-44.02) ** & $10.65(7.78-13.30)$ & $<0.001$ \\
\hline WBC $\left(\times 10^{9} / \mathrm{L}\right)$ & $5.29(3.57-5.99)$ & $5.05(3.59-5.53)$ & $5.25(3.86-6.02)$ & NS \\
\hline
\end{tabular}

Notes: Data are presented as median [interquartile range, (IQR)] and analysed using Mann-Whitney $U$-test or Wilcoxon ranked sum test; *P< 0.05 versus healthy controls; ** $\mathrm{P}<0.01$ versus healthy controls.

Abbreviations: IL, interleukin; IFN, interferon; TNF, tumor necrosis factor; CRP, C-reactive protein; ESR, erythrocyte sedimentation rate; WBC, white blood cell; NS, no significance.

\section{Correlation Between Serum Th 1/Th2/ ThI7 Cytokine Levels and General Inflammatory Biomarkers in Patients with Acute Brucellosis}

We evaluated the correlation between the levels of seven cytokines and those of general inflammatory biomarkers such as CRP, ESR, and WBC in patients with acute brucellosis. The results showed that the serum IL-2 and IL-6 levels correlated significantly with the CRP level during acute infection $(\mathrm{R}=0.5497, \mathrm{P}=0.0017 ; \mathrm{R}=0.5249, \mathrm{P}=$ 0.0029 , respectively) (Figure 2 and Table 3 ).

\section{Discussion}

Changes in the Th1/Th2/Th17 cytokine levels in patients with acute brucellosis during treatment have been scarcely investigated. In this study, we measured the levels of seven Th1/Th2/Th17 cytokines in serum of patients with acute brucellosis before treatment and after 6 weeks of successful treatment. Levels of most Th1 and Th2 cytokines elevated during acute infection and declined again after treatment. The TNF- $\alpha$ level significantly increased at 6 weeks after therapy. Finally, the levels of four cytokines, namely, IFN- $\gamma$, TNF- $\alpha$, IL-4, and IL-10, in the treated patients were still higher than those in healthy controls. This finding reflected a sustained Th1 and Th2 cytokine response in individuals cured of brucellosis, which may be potential therapeutic targets for improving long-term clinical outcomes in patients with acute brucellosis after treatment. Notably, the elevated serum IL-2, IFN- $\gamma$, and IL-10 levels decreased more significantly than those of other cytokines during treatment in individuals with acute infection, suggesting that the measurement of serum IL-2, IFN$\gamma$, and IL-10 levels may be helpful in assessment of the immune status and evaluation of treatment efficiency in acute brucellosis.

Human brucellosis is a systemic infectious disease, and the interaction between Brucella and the host defence systems mainly determines the development of chronic parasitism or pathogen clearance. ${ }^{6}$ Cell-mediated immune responses play an essential role in anti-Brucella protective immunity. ${ }^{7} \mathrm{CD}^{+} \mathrm{T}$ lymphocytes are the major immune regulatory cells in Brucella infection in humans and animal models ${ }^{9,12}$ and can differentiate into Th1, Th2, and Th17 cells depending on the cytokines they secrete. ${ }^{24,25}$ In recent years, new subsets of immune 
A

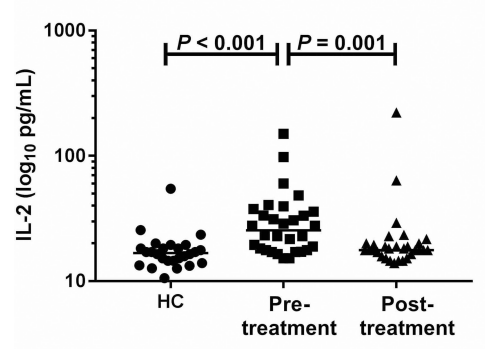

D

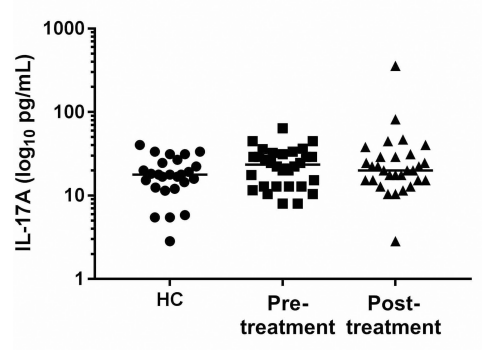

G

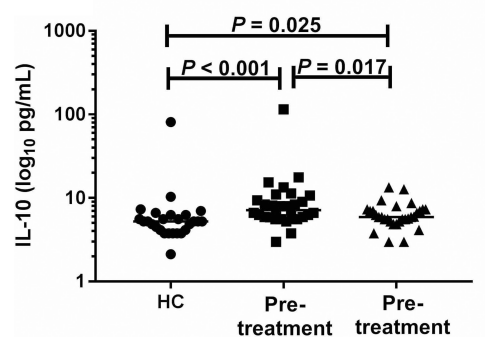

B

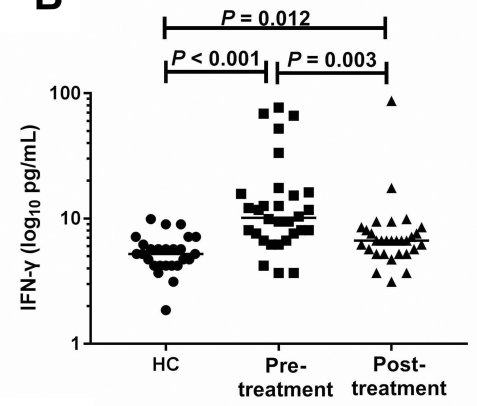

E

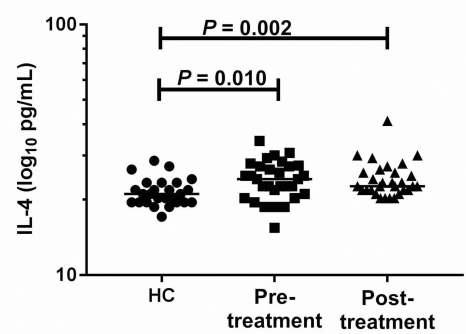

C

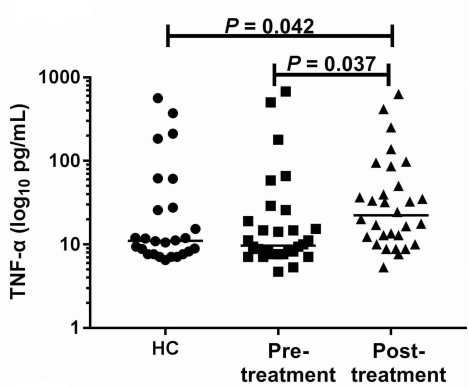

F

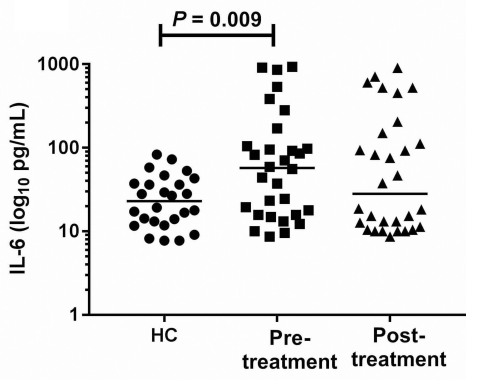

Figure I Serum IL-2, IFN- $\gamma$, TNF- $\alpha$, IL- I7A, IL-4, IL-6, and IL-I0 levels in patients with brucellosis before and after treatment and in healthy controls. Comparisons of the level of seven cytokines, including (A) IL-2, (B) IFN- $\gamma$, (C) TNF- $\alpha$, (D) IL-I7A, (E) IL-4, (F) IL-6, and (G) IL-I0 in patients with acute brucellosis before treatment (pretreatment) and at 6 weeks after treatment (post-treatment) and healthy controls $(\mathrm{HC})(\mathrm{n}=30,30$, and 26 , respectively) are shown. Values are expressed in Log 10 (pg/mL). Medians are indicated with horizontal lines. $P$ values were calculated using the Mann-Whitney U-test for differential analysis of controls and patients and the Wilcoxon matched-pair signed-rank test for comparisons of patients before and after treatment.

A

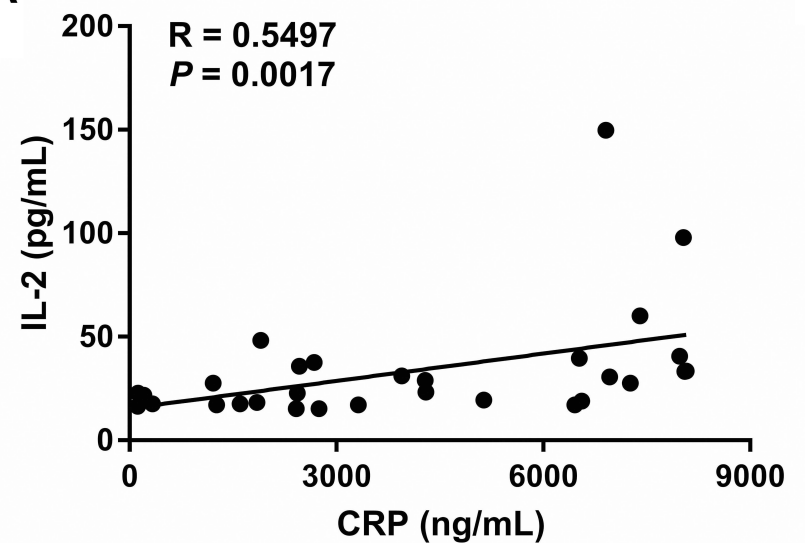

B

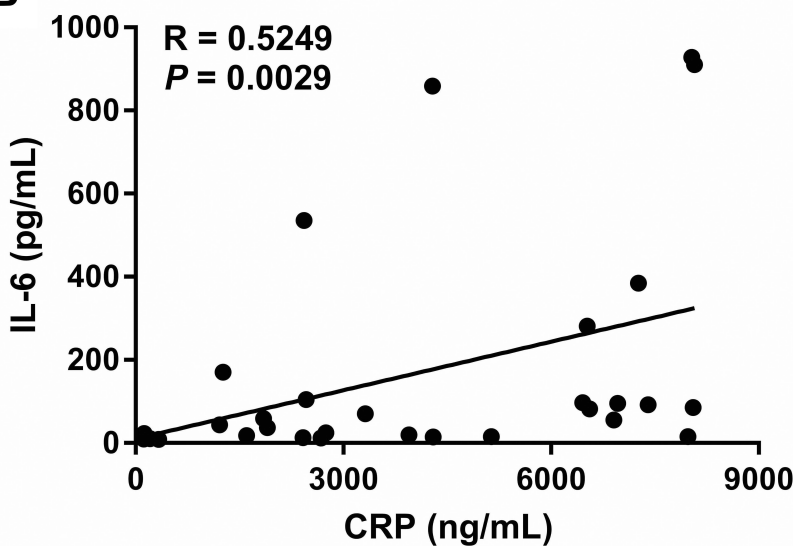

Figure 2 Correlations between selected serum ThI/Th2/ThI 7 cytokines and CRP. Spearman correlation coefficient (R) was used to evaluate correlations between CRP and serum IL-2 (A) and IL-6 (B) in patients with acute brucellosis $(n=30)$. 
Table 3 Correlations Between Serum Th1/Th2/Th 77 Cytokines with General Inflammatory Markers in Patients with Acute Brucellosis $(n=30)$

\begin{tabular}{|c|c|c|c|c|c|c|}
\hline \multirow[t]{2}{*}{ Cytokines } & \multicolumn{2}{|c|}{ CRP } & \multicolumn{2}{|c|}{ ESR } & \multicolumn{2}{|c|}{ WBC } \\
\hline & $\mathbf{R}$ & $P$ & $\mathbf{R}$ & $P$ & $\mathbf{R}$ & $P$ \\
\hline IL-2 & 0.550 & 0.002 & -0.207 & 0.273 & 0.146 & 0.443 \\
\hline IL-4 & -0.603 & $0.74 I$ & -0.260 & 0.165 & 0.210 & 0.265 \\
\hline IL-6 & 0.525 & 0.003 & -0.031 & $0.87 I$ & 0.301 & 0.106 \\
\hline IL-I0 & 0.308 & 0.097 & 0.045 & 0.814 & 0.148 & 0.435 \\
\hline TNF- $\alpha$ & 0.334 & 0.071 & -0.102 & 0.593 & -0.211 & 0.264 \\
\hline IFN- $\gamma$ & 0.111 & $0.56 \mathrm{I}$ & -0.074 & 0.696 & -0.152 & 0.423 \\
\hline IL-I7A & -0.152 & 0.423 & -0.058 & 0.762 & 0.248 & 0.186 \\
\hline
\end{tabular}

Notes: R, Spearman correlation coefficient.

Abbreviations: IL, interleukin; IFN, interferon; TNF, tumor necrosis factor; CRP, C-reactive protein; ESR, erythrocyte sedimentation rate; WBC, white blood cell.

regulatory cells, namely $\mathrm{Th} 9$, Th22, and follicular Th cells (Tfh), have been identified. ${ }^{26-28}$ Among the subsets of $\mathrm{CD}^{+} \mathrm{T}$ lymphocytes, Th1 cells are known to be critical for the clearance of Brucella, and Th2 cells are involved in susceptibility to Brucella and infection persistence. ${ }^{7,8,10}$ Coordination and balance of cytokines, particularly in Th1 and Th2 immune responses, may contribute to pathogenesis or resistance to Brucella. Therefore, measurement of these cytokines produced in the circulatory system may be helpful in evaluating the degree of immune activation and the extent of immune response during infection and therapy for treatment monitoring in brucellosis.

In this study, the significantly elevated serum levels of IFN- $\gamma$ and IL-2 represented a Th1 immune pattern in acute brucellosis. The production of IFN- $\gamma$ has been reported to be a defence mechanism against Brucella infection in the murine model. ${ }^{10,11}$ Our results are consistent with previous results, indicating that the serum IFN- $\gamma$ levels increase during acute infection and decline with treatment. ${ }^{29-31}$ IL-2 not only promotes cellular immune response to antigen but also plays pivotal roles in homeostasis of $\mathrm{CD}^{+}$ T-cell subsets, thereby mediating tolerance and restricting inappropriate immune reactions. ${ }^{32}$ Consistent with our results, a previous study reported that significantly elevated IL-2 levels declined during treatment in acute brucellosis. $^{31}$ TNF- $\alpha$ was established to enhance the ongoing Th1 response, causing clearance of the pathogen during Brucella infection. ${ }^{33}$ Notably, in our patients, the TNF- $\alpha$ levels were unchanged during acute infection but significantly elevated during treatment, suggesting the restoration of protective immune response in hosts in some way. A previous study reported that the increased serum TNF- $\alpha$ levels significantly declined in patients following treatment; ${ }^{31}$ the inconsistencies of these findings with our finding may be due to differences in the infection status of patients, therapeutic regimen, and cytokine examination method. Furthermore, Lin stated that the serum TNF- $\alpha$ level was elevated in acute brucellosis, which is inconsistent with the finding of our study; the possible reason for the inconsistency remains unclear and needs further evaluation. ${ }^{29}$ In addition, the increased levels of cytokines such as IL-4, IL-6, and IL-10 in the serum of untreated patients in this study highlighted a simultaneous Th1 and Th2 response, suggesting that the immune response to Brucella in these patients did not polarise as in inbred murine models or humans reported in some studies. $^{12,16,18}$ This result may support our previous study results indicating elevated percentages of $\mathrm{Th} 1$ and $\mathrm{Th} 2$ cells in patients with acute brucellosis. ${ }^{17}$ Th17 cells are induced by IL-6, IL-21, and TGF- $\beta$, and IL-17A is the major effector molecule of Th17 cells. ${ }^{24}$ IL-17A is a proinflammatory cytokine that promotes the protective immune response against bacterial infections but mediates tissue damage. ${ }^{34,35}$ Reports have shown that pathologies such as airway remodelling are related to high IL-17A levels in the serum of patients with pulmonary tuberculosis. ${ }^{36}$ Therefore, it seemed that IL-17A may be related to extensive immunopathological consequences in intracellular bacterial infection. The change in the serum IL-17A level was found to be nonsignificant in this study, which suggested that the patients in our study did not exhibit tissue damage. This result is inconsistent with those of two previous studies, which have reported that the IL-17A level increases in patients during acute infection. $^{18,29}$ In Lin's study, $46.0 \%$ patients with acute brucellosis had brucellosis arthritis and lumbar disc hyperplasia, which may be the major factors for elevation of the IL-17A level. ${ }^{29}$ In addition, the difference between the results of our study and Zheng's study may be partly related to the disease duration [shorter time (1-10 weeks) in our study vs $1-26$ weeks in Zhang's study]. ${ }^{18}$ Thus, our findings underlined the importance of early diagnosis and prompt initiation of antibiotic therapy for acute brucellosis, although further research is needed to confirm the present study findings.

In addition to the pro-inflammatory response, the antiinflammatory responses were stimulated during acute Brucella infection in our study. Th2 cytokines such as IL-4 and IL-6 contribute to B-cell differentiation and 
switching of antibody isotypes due to their antiinflammatory mechanisms; in addition, IL-4 plays a role in suppression of pro-inflammatory and cellular immune responses, which are associated with impaired protective immune response. ${ }^{20,37,38}$ Our finding indicating the elevated IL-4 and IL-6 levels in acute brucellosis is consistent with that of a previous report. ${ }^{29}$ Among our patients, the increase in the level of the anti-inflammatory cytokine IL10 was observed in acute infection, which indicated that IL-10 was induced to ensure homeostasis in patients during infection; the finding is consistent with those of previous studies. ${ }^{29,39,40}$ Xavier et al reported that a lack of IL10 production by $\mathrm{T}$ cells resulted in an increased function to control B. abortus infection while inducing elevated expression of pro-inflammatory cytokines in a murine model. ${ }^{13}$ Moreover, its ability to resist further proinflammatory responses was supported by reduced levels of pro-inflammatory cytokines during our patients' treatment. On the other hand, these mechanisms can cause defective host immune response and diminished elimination of the bacteria; however, they could also prevent excessive immune responses and reduce tissue damage. ${ }^{41}$

In this study, we demonstrated that the levels of four cytokines (the pro-inflammatory cytokines IFN- $\gamma$ and TNF- $\alpha$ and the anti-inflammatory cytokines IL-4 and IL10) were high in patients even after treatment. These results strongly suggested that the Th1 and Th2 cytokine responses remained after completion of adequate treatment, even though clinical symptoms and signs relatively disappeared and the CRP level and ESR normalised. Considering residual monocyte activation reported in our previous study, we speculate that the residual Th1 and Th2 cytokine responses may be related to persistent monocyte/ macrophage activation. Besides, these results are consistent with that of a previous study, which reported that the serum Th1 cytokine levels decreased with treatment but only partially reverted at follow-up. ${ }^{31}$ Similar results were reported in two studies indicating that the Th1 response remained higher within 12 months of treatment in convalescent patients with brucellosis than in controls. ${ }^{18,19}$ In addition, among children with brucellosis, Galanakis found that the increased serum IL-4 levels in the acute stage were still high during convalescence as compared with controls, suggesting a residual Th2 activation in children cured of brucellosis. ${ }^{20}$ Similarly, residual immune activation was present in HIV-infected individuals after HAART-induced HIV suppression, although the level of several biomarkers of T-cell activation returned to normal. ${ }^{42,43}$ Moreover, our data revealed that during acute infection, the serum levels of IL-2 and IL-6 positively correlated with the acute phase reactant CRP, a nonspecific indicator for severity of inflammatory diseases, ${ }^{44,45}$ reflecting that IL-2 and IL- 6 might be the candidate biomarkers for disease severity. After treatment, the correlation between the levels of seven cytokines with general inflammatory biomarkers was not assessed because the CRP level and ESR were normalised in the patients. In addition, we compared the levels of the seven cytokines and common inflammatory markers between patients with positive and negative blood culture and no significant difference was found.

Despite the novel findings of our study, the study has some limitations. First, our study was mono-centric with relatively smaller sample size, and the follow-up period was restricted. Further large multi-centre studies with longer follow-up period are warranted. Second, we enrolled only the patients with acute brucellosis in the current study; we aim to include patients in the subacute and chronic phases in our further study.

\section{Conclusion}

This study demonstrated that the serum levels of most Th1 and Th2 cytokines were significantly high during acute brucellosis but declined gradually with effective and adequate treatment. Moreover, the serum Th1 and Th2 cytokine levels were not normalised despite clinical recovery, indicating that residual Th1 and Th2 cytokine responses were present in the patients cured of acute brucellosis. These residual immune responses could represent a therapeutic opportunity that may improve the long-term clinical outcomes in patients with acute brucellosis after treatment. In addition, the elevated serum IL-2, IFN- $\gamma$, and IL-10 levels decreased more significantly than those of other cytokines during treatment in individuals with acute brucellosis, suggesting that the measurement of serum IL-2, IFN- $\gamma$, and IL-10 levels may be useful for immune status assessment and monitoring of treatment responses in acute brucellosis; however, there is a need for investigations with more follow-up points and larger numbers of patients to further confirm the point.

\section{Abbreviations}

ELISA, enzyme-linked immunosorbent assay; IL-2, interleukin 2; IFN- $\gamma$, interferon gamma; TNF- $\alpha$, tumour necrosis factor alpha; IL-4, interleukin 4; IL-6, interleukin 6; IL10, interleukin-10; IL-17A, interleukin-17A; Th, T helper 
cells; CRP, C-reactive protein; ESR, erythrocyte sedimentation rate; $\mathrm{WBC}$, white blood cell.

\section{Ethics Approval and Informed Consent}

The study was approved by the Ethics Committee of Beijing Ditan Hospital of Capital Medical University (Approval number: 2018-052-01) and conformed to the Declaration of Helsinki. In addition, written informed consent was obtained from each subject.

\section{Consent for Publication}

Written informed consent for publication was obtained from all participants.

\section{Acknowledgments}

The authors are grateful to technicians from the clinical laboratory and the medical staff of the Department of Infectious Diseases of Beijing Di Tan Hospital for their valuable contribution in sample collection as well as to all of the healthy controls. The authors thank Dr. Yali Wu for language improvement and revision of the manuscript. We would like to thank Editeg (www.editeg.com) for English language editing.

\section{Funding}

This study was supported by Fund Grant of Open Project of Beijing Key Laboratory of Emerging Infectious Diseases (Grant No. DTKF201804).

\section{Disclosure}

The authors report no conflicts of interest in this work.

\section{References}

1. Dean AS, Crump L, Greter H, et al. Global burden of human brucellosis: a systematic review of disease frequency. PLoS Negl Trop Dis. 2012;6(10):e1865. doi:10.1371/journal.pntd.0001865

2. Jiang H, O'Callaghan D, Ding JB. Brucellosis in China: history, progress and challenge. Infect Dis Poverty. 2020;9(1):55 doi: 10.1186/s40249-020-00673-8

3. Amjadi O, Rafiei A, Mardani M, et al. A review of the immunopathogenesis of Brucellosis. Infect Dis. 2019;51(5):321-333. doi:10.1080/ 23744235.2019.1568545

4. Editorial Board of Chinese Journal of Infectious Diseases. Expert consensus on diagnosis and treatment of brucellosis. Chin J Infect Dis. 2017;35(12):705-710.

5. Bosilkovski M, Keramat F, Arapović J. The current therapeutical strategies in human brucellosis. Infection. 2021;49(5):823-832. doi:10.1007/s15010-021-01586-w
6. de Figueiredo P, Ficht TA, Rice-Ficht A, et al. Pathogenesis and immunobiology of brucellosis: review of Brucella-host interactions. Am J Pathol. 2015;185(6):1505-1517. doi:10.1016/j.ajpath.2015.03.003

7. Skendros P, Pappas G, Boura P. Cell-mediated immunity in human brucellosis. Microbes Infect. 2011;13(2):134-142. doi:10.1016/j. micinf.2010.10.015

8. Yingst S, Hoover DL. T cell immunity to brucellosis. Crit Rev Microbiol. 2003;29(4):313-331. doi:10.1080/713608012

9. Cannella AP, Tsolis RM, Liang L, et al. Antigen-specific acquired immunity in human brucellosis: implications for diagnosis, prognosis, and vaccine development. Front Cell Infect Microbiol. 2012;2:1. doi:10.3389/fcimb.2012.00001

10. Goenka R, Parent MA, Elzer PH, et al. B cell-deficient mice display markedly enhanced resistance to the intracellular bacterium Brucella abortus. J Infect Dis. 2011;203(8):1136-1146. doi:10.1093/infdis/ jiq171

11. High KP, Prasad R, Marion CR, et al. Outcome and immune responses after Brucella abortus infection in young adult and aged mice. Biogerontology. 2007;8(5):583-593. doi:10.1007/s10522-0079106-6

12. Vitry MA, De Trez C, Goriely S, et al. Crucial role of gamma interferon-producing $\mathrm{CD} 4+\mathrm{Th} 1$ cells but dispensable function of CD8+ T cell, B Cell, Th2, and Th17 responses in the control of Brucella melitensis infection in mice. Infect Immun. 2012;80 (12):4271-4280. doi:10.1128/IAI.00761-12

13. Xavier MN, Winter MG, Spees AM, et al. CD4+ T cell-derived IL-10 promotes Brucella abortus persistence via modulation of macrophage function. PLoS Pathog. 2013;9(6):e1003454. doi:10.1371/journal. ppat. 1003454

14. Roop RM, Barton IS, Hopersberger D, et al. Uncovering the hidden credentials of Brucella virulence. Microbiol Mol Biol Rev. 2021;85 (1):e00021-19.

15. Vitry MA, Hanot MD, De Trez C, et al. Humoral immunity and CD4 + Th1 cells are both necessary for a fully protective immune response upon secondary infection with Brucella melitensis. J Immunol. 2014;192(8):3740-3752. doi:10.4049/jimmunol.1302561

16. Rafiei A, Ardestani SK, Kariminia A, et al. Dominant Th1 cytokine production in early onset of human brucellosis followed by switching towards Th2 along prolongation of disease. J Infect. 2006;53 (5):315-324. doi:10.1016/j.jinf.2005.11.024

17. Sun HL, Ma CJ, Du XF, et al. Soluble IL-2R $\alpha$ correlates with imbalances of Th1/Th2 and Tc1/Tc2 cells in patients with acute brucellosis. Infect Dis Poverty. 2020;9(1):92. doi:10.1186/s40249020-00699-y

18. Zheng R, Xie S, Zhang Q, et al. Circulating Th1, Th2, Th17, Treg, and PD-1 levels in Patients with Brucellosis. J Immunol Res. 2019;2019:3783209. doi:10.1155/2019/3783209

19. Xu G, Zhang P, Dang R, et al. Dynamic changes of Th1 cytokines and the clinical significance of the IFN- $\gamma / \mathrm{TNF}-\alpha$ ratio in acute Brucellosis. Mediators Inflamm. 2019;2019:5869257. doi:10.1155/ 2019/5869257

20. Galanakis E, Makis A, Bourantas KL, et al. Interleukin-3 and interleukin-4 in childhood brucellosis. Infection. 2002;30(1):33-34. doi:10.1007/s15010-002-2039-8

21. Sun H, Jiang R, Han B, et al. Serum levels of soluble CD163 and soluble CD14 following antibiotic therapy of patients with acute brucellosis. J Infect Dev Ctries. 2019;13(08):714-719. doi:10.3855/ jidc. 10602

22. Shostak E, Krause I, Dagan A, et al. Is serum CRP level a reliable inflammatory marker in pediatric nephrotic syndrome. Pediatr Nephrol. 2016;31(8):1287-1293. doi:10.1007/s00467-016-3328-2

23. Williams A, Steffens F, Reinecke C, et al. The Th1/Th2/Th17 cytokine profile of HIV-infected individuals: a multivariate cytokinomics approach. Cytokine. 2013;61(2):521-526. doi:10.1016/j. cyto.2012.11.006 
24. Korn T, Bettelli E, Oukka M, et al. IL-17 and Th17 cells. Annu Rev Immunol. 2009;27(1):485-517. doi:10.1146/annurev. immunol.021908.132710

25. Raphael I, Nalawade S, Eagar TN, et al. T cell subsets and their signature cytokines in autoimmune and inflammatory diseases. Cytokine. 2015;74(1):5-17. doi:10.1016/j.cyto.2014.09.011

26. Staudt V, Bothur E, Klein M, et al. Interferon-regulatory factor 4 is essential for the developmental program of $\mathrm{T}$ helper 9 cells. Immunity. 2010;33(2):192-202. doi:10.1016/j.immuni.2010.07.014

27. Scriba TJ, Kalsdorf B, Abrahams DA, et al. Distinct, specific IL-17and IL-22-producing CD4+ $\mathrm{T}$ cell subsets contribute to the human anti-mycobacterial immune response. $J$ Immunol. 2008;180 (3):1962-1970. doi:10.4049/jimmunol.180.3.1962

28. Crotty S. Follicular helper CD4 T cells (TFH). Annu Rev Immunol. 2011;29(1):621-663. doi:10.1146/annurev-immunol-031210-101400

29. Lin ZQ, Lin GY, He WW, et al. IL-6 and INF-gamma levels in patients with brucellosis in severe epidemic region, Xinjiang, China. Infect Dis Poverty. 2020;9(1):47. doi:10.1186/s40249-02000666-7

30. Rahmanpour M, Keramat F, Jourghasemi S, et al. Direct correlation between Th1 and Th17 responses in immunity to Brucella infection. Microbes Infect. 2019;21(10):441-448. doi:10.1016/j.micinf.2 019.05 .002

31. Rodríguez-Zapata M, Matías MJ, Prieto A, et al. Human brucellosis is characterized by an intense Th1 profile associated with a defective monocyte function. Infect Immun. 2010;78(7):3272-3279. doi:10.1128/IAI.01385-09

32. Liao W, Lin JX, Leonard WJ. Interleukin-2 at the crossroads of effector responses, tolerance, and immunotherapy. Immunity. 2013;38(1):13-25. doi:10.1016/j.immuni.2013.01.004

33. Dornand J, Gross A, Lafont V, et al. The innate immune response against Brucella in humans. Vet Microbiol. 2002;90(1-4):383-394. doi:10.1016/S0378-1135(02)00223-7

34. Cooper AM. IL-17 and anti-bacterial immunity: protection versus tissue damage. Eur J Immunol. 2009;39(3):649-652. doi:10.1002/ eji.200839090

35. Brodlie M, McKean MC, Johnson GE, et al. Raised interleukin-17 is immunolocalised to neutrophils in cystic fibrosis lung disease. Eur Respir J. 2011;37(6):1378-1385. doi:10.1183/09031936.00067110
36. Torrado E, Cooper AM. IL-17 and Th17 cells in tuberculosis. Cytokine Growth Factor Rev. 2010;21(6):455-462. doi:10.1016/j. cytogfr.2010.10.004

37. Tanaka T, Narazaki M, Kishimoto T. IL-6 in inflammation, immunity, and disease. Cold Spring Harb Perspect Biol. 2014;6(10):a016295. doi:10.1101/cshperspect.a016295

38. Li Z, Zhang H, Zhang J, et al. Brucella abortus phosphoglyceromutase and dihydrodipicolinate reductase induce Th1 and Th2-related immune responses. World J Microbiol Biotechnol. 2018;34(2):22. doi:10.1007/s11274-017-2405-4

39. Sun HL, Du XF, Tang YX, et al. Impact of immune checkpoint molecules on FoxP3(+) Treg cells and related cytokines in patients with acute and chronic brucellosis. BMC Infect Dis. 2021;21(1):1025. doi:10.1186/s12879-021-06730-3

40. Ouyang W, Rutz S, Crellin NK, et al. Regulation and functions of the IL-10 family of cytokines in inflammation and disease. Annu Rev Immunol. 2011;29(1):71-109. doi:10.1146/annurev-immunol-03121 $0-101312$

41. Bedke T, Muscate F, Soukou S, et al. Title: IL-10-producing T cells and their dual functions. Semin Immunol. 2019;44:101335. doi:10.1016/j.smim.2019.101335

42. Wada NI, Jacobson LP, Margolick JB, et al. The effect of HAART-induced HIV suppression on circulating markers of inflammation and immune activation. AIDS. 2015;29(4):463-471. doi:10.1097/QAD.0000000000000545

43. Burdo TH, Lentz MR, Autissier P, et al. Soluble CD163 made by monocyte/macrophages is a novel marker of HIV activity in early and chronic infection prior to and after anti-retroviral therapy. $J$ Infect Dis. 2011;204(1):154-163. doi:10.1093/infdis/jir214

44. Prins HJ, Duijkers R, van der Valk P, et al. CRP-guided antibiotic treatment in acute exacerbations of COPD in hospital admissions. Eur Respir J. 2019;53(5):1802014.

45. Wilson D, Moosa MS, Cohen T, et al. Evaluation of tuberculosis treatment response with serial C-reactive protein measurements. Open Forum Infect Dis. 2018;5(11):ofy253. doi:10.1093/ofid/ofy253
Infection and Drug Resistance

\section{Publish your work in this journal}

Infection and Drug Resistance is an international, peer-reviewed openaccess journal that focuses on the optimal treatment of infection (bacterial, fungal and viral) and the development and institution of preventive strategies to minimize the development and spread of resistance. The journal is specifically concerned with the epidemiology of antibiotic resistance and the mechanisms of resistance development and diffusion in both hospitals and the community. The manuscript management system is completely online and includes a very quick and fair peerreview system, which is all easy to use. Visit http://www.dovepress.com/ testimonials.php to read real quotes from published authors. 